\title{
Real-space cluster dynamical mean-field theory: Center-focused extrapolation on the one- and two particle-levels
}

\author{
Marcel Klett $\odot,{ }^{1, *}$ Nils Wentzell, ${ }^{2}$ Thomas Schäfer $\odot,{ }^{3,4}$ Fedor Simkovic, IV,, 3 Olivier Parcollet,,${ }^{2,5}$ Sabine Andergassen $\odot,{ }^{1}$ \\ and Philipp Hansmann (1) 6,7 \\ ${ }^{1}$ Institut für Theoretische Physik and Center for Quantum Science, Universität Tübingen, Auf der Morgenstelle 14, 72076 Tübingen, Germany \\ ${ }^{2}$ Center for Computational Quantum Physics, Flatiron institute, Simons Foundation, 162 5th Ave., New York, 10010 New York, USA \\ ${ }^{3}$ Collège de France, 11 place Marcelin Berthelot, 75005 Paris, France \\ ${ }^{4}$ CPHT, CNRS, École polytechnique, IP Paris, F-91128 Palaiseau, France \\ ${ }^{5}$ Université Paris-Saclay, CNRS, CEA, Institut de physique théorique, 91191, Gif-sur-Yvette, France \\ ${ }^{6}$ Max-Planck-Institut für Chemische Physik fester Stoffe, Nöthnitzerstr. 40, 01187 Dresden, Germany \\ ${ }^{7}$ Department of Physics, University of Erlangen-Nuremberg, 91058 Erlangen, Germany
}

(Received 11 March 2020; revised 14 July 2020; accepted 27 August 2020; published 23 September 2020)

\begin{abstract}
We revisit the cellular dynamical mean-field theory (CDMFT) for the single-band Hubbard model on the square lattice at half filling, reaching real-space cluster sizes of up to $9 \times 9$ sites. Using benchmarks against direct lattice diagrammatic Monte Carlo at high temperature, we show that the self-energy obtained from a cluster center-focused extrapolation converges faster with the cluster size than the periodization schemes previously introduced in the literature. The same benchmark also shows that the cluster spin susceptibility can be extrapolated to the exact result at large cluster size, even though its spatial extension is larger than the cluster size.
\end{abstract}

DOI: 10.1103/PhysRevResearch.2.033476

\section{INTRODUCTION}

Even after decades of intense research, the single band Hubbard model in finite dimensions larger than one remains an unsolved cornerstone paradigm in theoretical solid state physics. As a nonperturbative technique dynamical mean-field theory (DMFT) [1-3] led to a significant leap forward as it provided an approximation which is nowadays widely used to treat material realistic variants of the Hubbard model [4]. In DMFT, the lattice problem is mapped to a self-consistent auxiliary quantum impurity model, leading to a local approximation of the self-energy.

Single site DMFT has led to many successes, e.g., a description of the Mott-Hubbard metal-to-insulator transition (MIT) from a weak coupling metallic over to a strongly correlated Fermi liquid and eventually Mott insulating state [3,5] within a single theoretical framework. The locality of the self-energy is however a major limitation for some of the most interesting problems as, for example, cuprate high$T_{c}$ superconductivity [6-9] where the pseudogap phase is characterized by a strong node-antinode differentiation. Note, however, that in material-realistic multiorbital models, even

\footnotetext{
*marcel.klett@uni-tuebingen.de
}

Published by the American Physical Society under the terms of the Creative Commons Attribution 4.0 International license. Further distribution of this work must maintain attribution to the author(s) and the published article's title, journal citation, and DOI. Open access publication funded by the Max Planck Society. local DMFT self-energies can lead to $\mathbf{k}$ dependent effects in the spectral function $A(\mathbf{k}, \omega)$ due to orbital mixing as, e.g., shown in Ref. [10]. Quantum critical behavior [11-13] in low dimensions and/or at low temperatures is another example where the physics is dominated by nonlocal correlations beyond the mean-field description of spatial fluctuations within DMFT.

In recent years, several methods have been introduced to overcome the locality of the self-energy approximation in DMFT, for a review on complementary efforts in the weak coupling regime see [14]. Firstly, cluster extensions of DMFT, in reciprocal space (DCA) [15-18], in real space (CDMFT) [19-21], or in its first "nested" form [3,22,23]. This transformed DMFT into a controlled method. The control parameter is the size of the cluster, which determines also the resolution of the approximation in momentum space. These approaches have found extensive use, e.g., in the study of the Hubbard model [24-41]. Secondly, several diagrammatic extensions [42-58] have been proposed to obtain a better resolution in momentum or a better convergence than cluster methods.

In this paper, we focus on the real space CDMFT method [20]. While large cluster sizes were studied in DCA up to convergence and compared to exact methods like diagrammatic Monte Carlo in some parameter regimes, e.g., Refs. [59,60], large CDMFT clusters have not received the same attention. The central issue is that CDMFT breaks translational invariance by definition for any finite cluster. One therefore needs to use reperiodization schemes to restore the translation symmetry of the lattice self-energy when approximating it from the cluster self-energy [20]. We note in passing that such a 
reperiodization is usually performed only for the converged result as it tends to destabilize the calculation when performed during the self consistency loop. Several ways of reperiodization been discussed in the literature $[26,61,62]$.

In this work, we solve large CDMFT clusters of up to $9 \times 9$ sites for the half-filled Hubbard model, and benchmark their convergence against exact results obtained with diagrammatic Monte Carlo (DiagMC) [63-65] in its connected determinant formulation (CDet [66] for one particle reducible quantities and $\Sigma$ DDMC [67-69] for one particle irreducible quantities).

We show that if we use a center-focused extrapolation (CFE) of converged results for increasingly large clusters to approximate the lattice self-energy, instead of averaging over a whole single cluster as done in previous works, we obtain a much better approximation. The improvement of CFE with respect to previous schemes is quantified by its excellent agreement with the DiagMC result at moderate temperature. We further present results at lower temperature $T$ and larger $U$ than the range currently accessible with diagrammatic Monte Carlo methods.

The paper is organized as follows. In Sec. II, we introduce the 2D Hubbard model and present the CDMFT formalism. We then present in Sec. III the center-focused extrapolation technique, and benchmark it against DiagMC. In Sec. IV, we discuss the spin-spin correlation function and finally summarize our results in $\mathrm{Sec}$. V.

\section{MODEL AND METHOD}

We study the 2D single-orbital Hubbard model on the square lattice

$$
H=-t \sum_{<i, j>, \sigma} c_{i, \sigma}^{\dagger} c_{j, \sigma}+\sum_{i} U \hat{n}_{i, \uparrow} \hat{n}_{i, \downarrow}-\mu \sum_{i, \sigma} \hat{n}_{i, \sigma},
$$

where $c_{i, \sigma}^{\dagger}\left(c_{i, \sigma}\right)$ denotes the creation (annihilation) operator for an electron with spin $\sigma$ on site $i$, with the density operator $\hat{n}_{i, \sigma}=c_{i, \sigma}^{\dagger} c_{i, \sigma}$ and the chemical potential $\mu . t$ and $U$ are nearest-neighbor hopping amplitude and onsite Hubbard repulsion, respectively. We study the half-filled case, which corresponds to $\mu=U / 2$. For a single-orbital unit cell, the electronic dispersion relation in momentum space reads $\varepsilon(\mathbf{k})=-2 t\left(\cos k_{x}+\cos k_{y}\right)$.

In the following, we compute propagators of single- and two-particle excitations in the CDMFT approximation [20]. CDMFT is a DMFT approximation on the superlattice made of real space clusters on the original Bravais lattice [20], where the cluster sites within the supercell play the role of orbitals. Hence, the Green's functions and self-energy contains intersites elements. They are computed using a self-consistent auxiliary quantum impurity model, which is solved by a continuous-time quantum Monte-Carlo solver using an interaction expansion [70], implemented with the TRIQS library [71].

For the present study we consider square clusters of up to $N \times N=9 \times 9$ atoms (see Fig. 1 for a schematic illustration). Technically, the equations for the Green's functions and selfenergies are identical to those of a multiorbital (single-site) DMFT with a density-density interaction. The local lattice Green's function in Matsubara frequencies is computed by
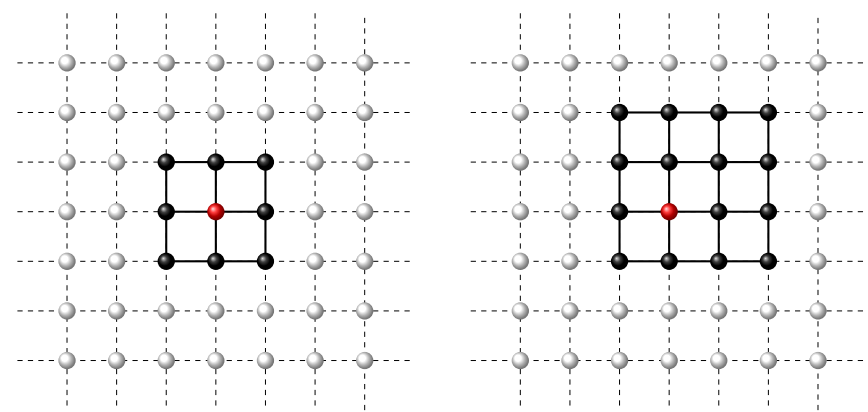

FIG. 1. Square clusters with $N \times N$ lattice sites. The left panel shows the $3 \times 3$ and the right one the $4 \times 4$ cluster, with the central site $\mathbf{R}_{c}$ in red.

integrating over the reduced Brillouin zone (RBZ) of the superlattice:

$G_{i j}^{\mathrm{loc}}\left(\mathrm{i} \omega_{n}\right)=\sum_{\mathbf{k} \in \mathrm{RBZ}}\left[\left(\mathrm{i} \omega_{n}+\mu\right) \delta_{i j}-\tilde{\varepsilon}_{i j}(\mathbf{k})-\Sigma_{i j}\left(\mathrm{i} \omega_{n}\right)\right]^{-1}$,

with $i, j$ indexing cluster sites, supercell dispersion relation $\tilde{\varepsilon}_{i j}(\mathbf{k})$ (with $\mathbf{k} \in \mathrm{RBZ}$ ), and self-energy $\Sigma_{i j}\left(\mathrm{i} \omega_{n}\right)$. Here the self-energy is obtained in every iteration from the auxiliary impurity model $\Sigma_{i j}\left(\mathrm{i} \omega_{n}\right)=\Sigma_{i j}^{\mathrm{imp}}\left(\mathrm{i} \omega_{n}\right)$. The Weiss field is then calculated as [20]

$$
\left[\mathcal{G}^{0}\left(\mathrm{i} \omega_{n}\right)\right]_{i j}^{-1}=\left[G^{\mathrm{loc}}\left(\mathrm{i} \omega_{n}\right)\right]_{i j}^{-1}+\Sigma_{i j}^{\mathrm{imp}}\left(\mathrm{i} \omega_{n}\right) .
$$

The self-energy acquires intersite components. Hence it can capture correlation effects up to distances of about the linear size of the cluster.

The cluster self-energies break translational invariance for any finite cluster size. Transformation to lattice quantities in the Brillouin zone of the original lattice therefore requires restoring translational invariance for the Green's function, the self-energy, or its cumulants [19,20,25,26,61,72-74] (see also Appendix B).

\section{CENTER-FOCUSED EXTRAPOLATION}

In a very large cluster, we expect on general grounds the Green function at the center of the cluster to converge faster with cluster size than at the boundary. In this section, we use this insight to improve on the reperiodization within the CDMFT algorithm. We begin with the study of single-particle cluster quantities and analyze the convergence of the local and the nearest-neighbor self-energy with respect to cluster size. In a second step, we use these converged self-energies to approximate the lattice self-energy, and benchmark it against exact DiagMC results.

\section{A. Cluster quantities}

Let us first concentrate on the cluster Green's and spectral functions. In Fig. 2, we show site-resolved single particle spectral functions obtained from an $N \times N=8 \times 8$ CDMFT calculation for different values of $U$. Exploiting cluster symmetry, we show only the upper right quadrant of the 64 cluster sites, where equivalent spectra are plotted in the same color. The violation of the translational invariance is most 

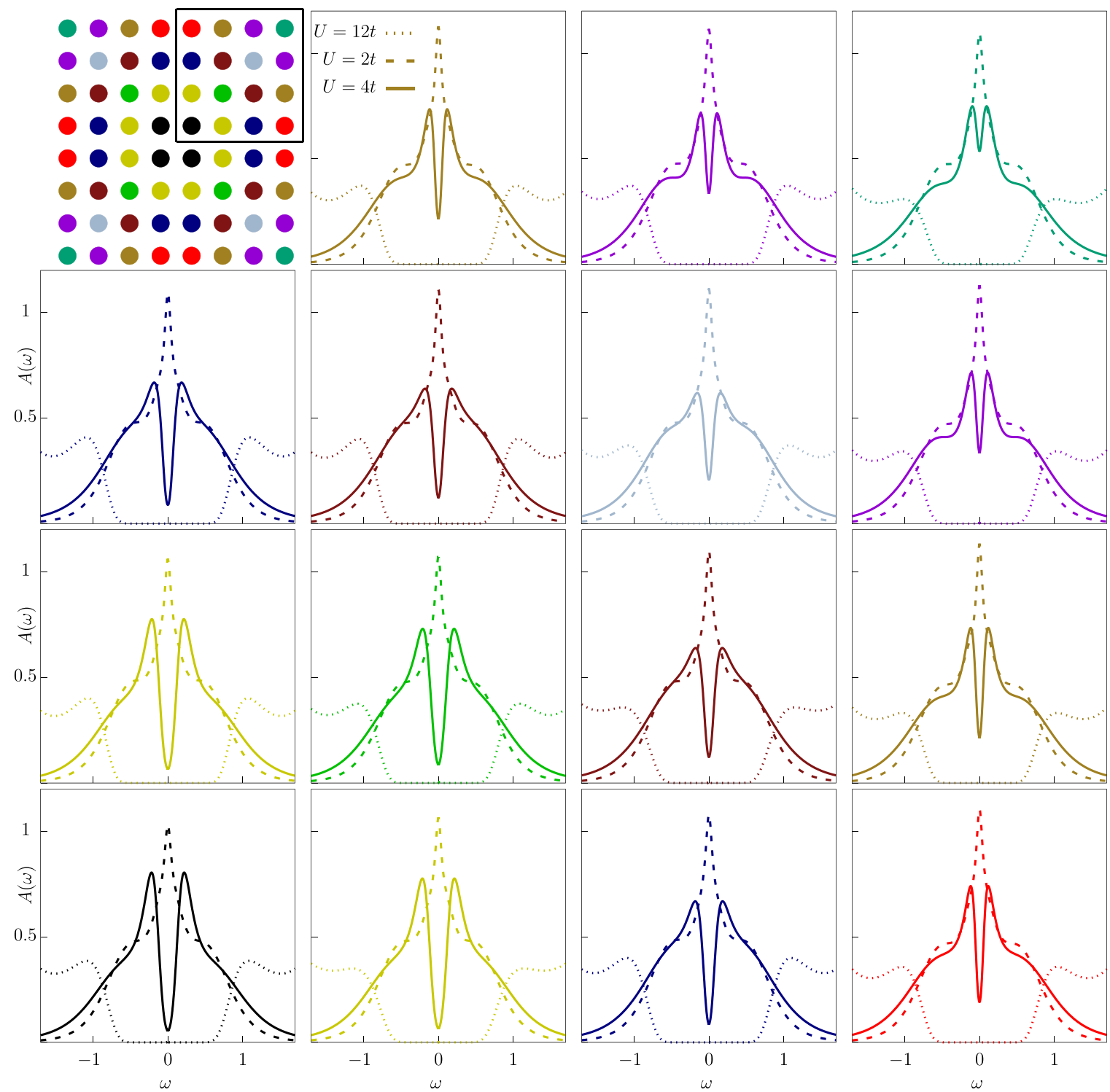

FIG. 2. Local spectral functions for $\beta t=12.5, N \times N=8 \times 8$ and different values of the interaction strength $U$. The shown panels present the upper right quadrant of our cluster (see upper left panel). There is a clear trend towards insulating behavior as we traverse the cluster from the border to the center.

pronounced for $U / t=4$ (solid lines) as can be seen by comparing the spectra in the center at $\mathbf{R}=(0,0)$ with those in the corners at $\mathbf{R}=(3,3)$. For $U / t=2$, all spectra exhibit a finite quasiparticle weight at $\epsilon_{F}$, while for $U / t=12$ they are all gaped.

In Fig. 3, we show the corresponding site-dependent value of $G(\mathbf{R}, \tau=\beta / 2)$. The spectra in the cluster center are generally more correlated/insulating than those at the edges/corners of the cluster. Given the fact that the exact solution has a critical value of $U_{c}=0$ at $T=0$, the single particle spectrum of the central site appears to be a better approximation than the average over the whole cluster (dashed line in Fig. 3).

\section{B. Convergence of cluster quantities}

We now study systematically the convergence of the onsite and first-neighbor self-energy with cluster size, and compare it to the exact lattice DiagMC result in the regime where it is available. In the left panels of Fig. 4, we plot the (purely

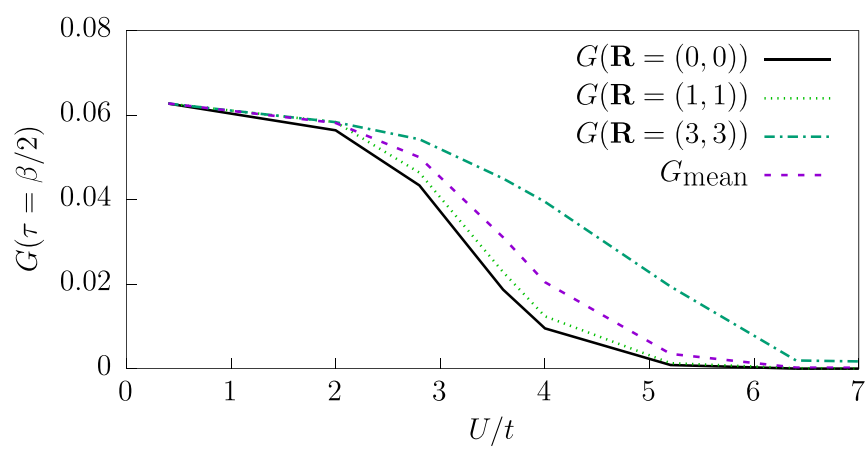

FIG. 3. Averaged (dashed) and site dependent $G(\tau=\beta / 2)$ as a function of $U / t$ with parameters identical to Fig. 2. 

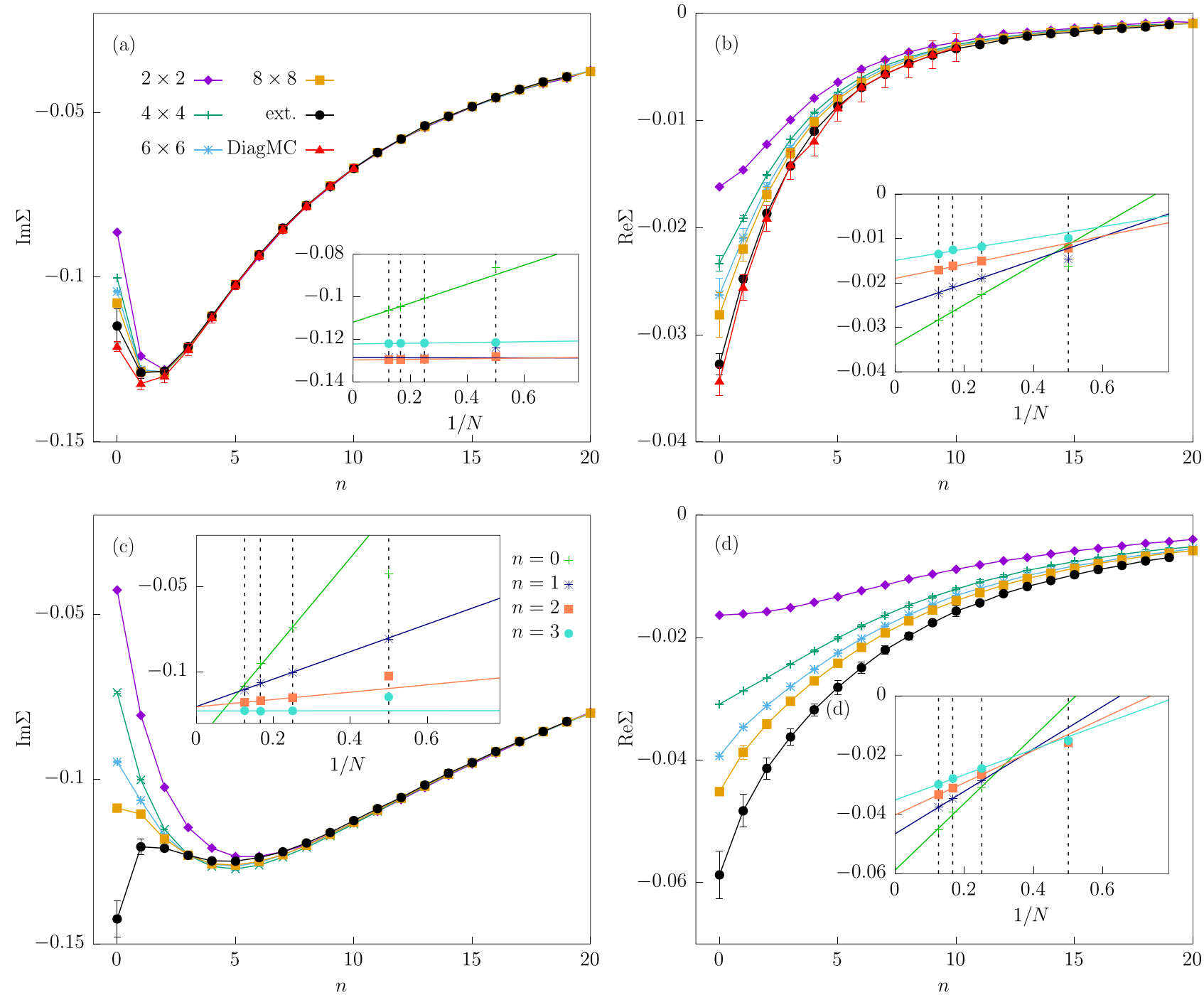

FIG. 4. Matsubara frequency dependent self-energy of the central site (a) and (c) at $\mathbf{R}=(0,0)$ and of the neighboring one (b) and (d) at $\mathbf{R}=(1,0)$, as obtained from CDMFT and DiagMC calculations for $U / t=4$ and $\beta t=5[$ (a) and (b)] as well as $\beta t=12.5$ [(c) and (d)]. The extrapolated self-energy is obtained by fitting the data with a function linear in $1 / N$ (excluding the $2 \times 2$ data), see insets for the fits from the first four Matsubara frequencies.

imaginary) onsite self-energies of the four inner-core sites of the even clusters as a function of Matsubara frequency for $U / t=4$ and two different temperatures. In the right panels, we plot the (purely real) nearest-neighbor self-energy corresponding to the intersite correlation between two of the innermost cluster sites. The overall cluster-size dependence is pronounced in all plots and stronger for the lower temperature. In the insets of Fig. 4, we show the linear extrapolation of the data at each Matsubara frequency as a function of $1 / N$. A linear extrapolation is possible, ${ }^{1}$ if we neglect the smallest cluster $N \times N=2 \times 2$. The extrapolated self-energies are plotted in black. The error bar combines errors from three

\footnotetext{
${ }^{1}$ According to spin-wave theory [88] finite-size corrections scale as $1 / N$, which may apply to the half-filled Hubbard model dominated by antiferromagnetic spin fluctuations.
}

sources: the QMC statistics in the quantum impurity solver, the convergence of the CDMFT self-consistency loop, and the extrapolation in $1 / N$.

At high temperature ( $\beta t=5$, upper panels of Fig. 4), we compare CDMFT results to DiagMC benchmark data for Matsubara frequencies $i \omega_{n}$ up to $n=10$ (red color). For both the onsite and nearest-neighbor self-energies, the extrapolated CDMFT results are compatible with the DiagMC data within error bars. For the onsite self-energy (upper left panel), the extrapolated (and DiagMC) self-energy is quite different from the largest considered cluster $N \times N=8 \times 8$, especially at the first $n=0$ Matsubara frequency. For the nearest-neighbor self-energy, the extrapolation effect extends to Matsubara frequencies as high as $n=10$.

At lower temperature ( $\beta t=12.5$, lower panels of Fig. 4), which is out of reach of the DiagMC algorithm, the effects of the extrapolation are even stronger and enhance the impact of the correlations. 


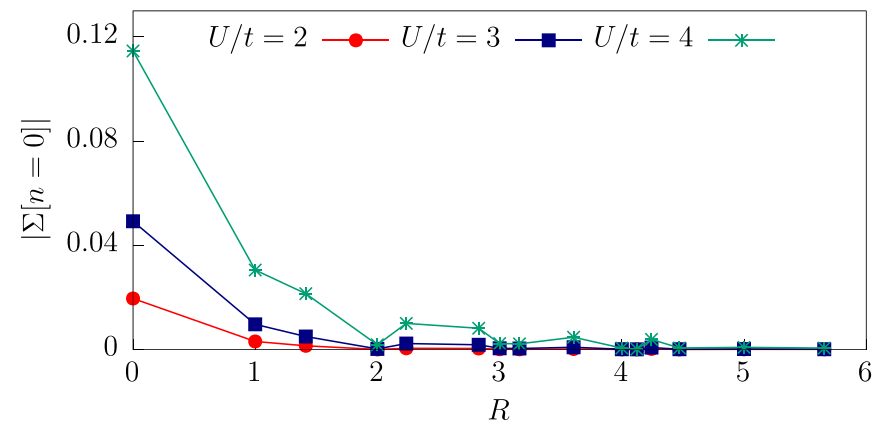

FIG. 5. Absolute value of the self-energy at the first Matsubara frequency $\Sigma\left(i \omega_{1}, \mathbf{R}\right)$ as a function of real-space distance $R=|\mathbf{R}|$ at $\beta t=5$ and different values of the interaction $U / t$.

\section{Center-focused extrapolation (CFE) of the self-energy}

After extrapolating the self-energy at the center of the cluster, we approximate the lattice self-energy in real space by a center-focused extrapolation (CFE) defined in the following.

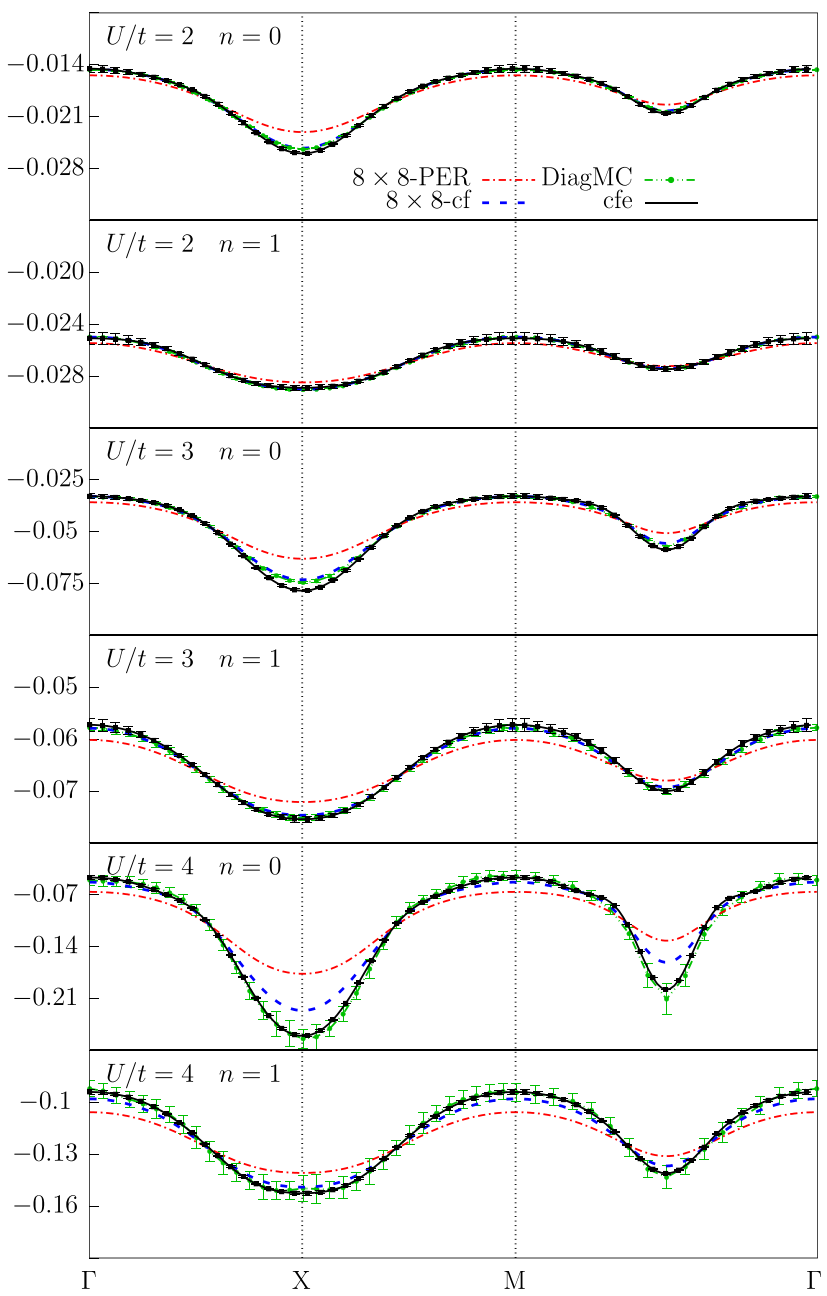

For each lattice displacement $\mathbf{R}$ with vector elements greater than or equal to zero, we take the large $N$ extrapolation of the cluster self-energy between the central site $\mathbf{R}_{\mathbf{c}}$ (cf. Fig. 1) and $\mathbf{R}+\mathbf{R}_{\mathbf{c}}$, i.e.,

$$
\Sigma_{\text {latt }}\left(\mathbf{R}, i \omega_{n}\right)=\Sigma_{\mathbf{R}+\mathbf{R}_{\mathbf{c}}, \mathbf{R}_{\mathbf{c}}}^{N \rightarrow \infty}\left(i \omega_{n}\right),
$$

For negative displacements we infer the value by using the rotational symmetry of the cluster. This procedure differs substantially from the conventional periodization, where averages over the whole cluster are performed [20].

In practice, given that we solve CDMFT only up to $N \times$ $N=9 \times 9$, our strategy is two-fold. For the short distances $\mathbf{R}$, we use the extrapolated self-energy values computed in the previous subsection. For the largest $\mathbf{R}$ however, where no $1 / N$ extrapolation is possible given the lack of data points, we use instead the value of the largest cluster. This approximation is justified because the self-energy in real space $\Sigma\left(i \omega_{n}, \mathbf{R}\right)$ decays on length scales smaller than our largest cluster. Indeed in Fig. 5, we show $\Sigma\left(i \omega_{1}, \mathbf{R}\right)$ for $\beta t=5$ and different values of the interaction $U / t$. Even for the largest interaction value

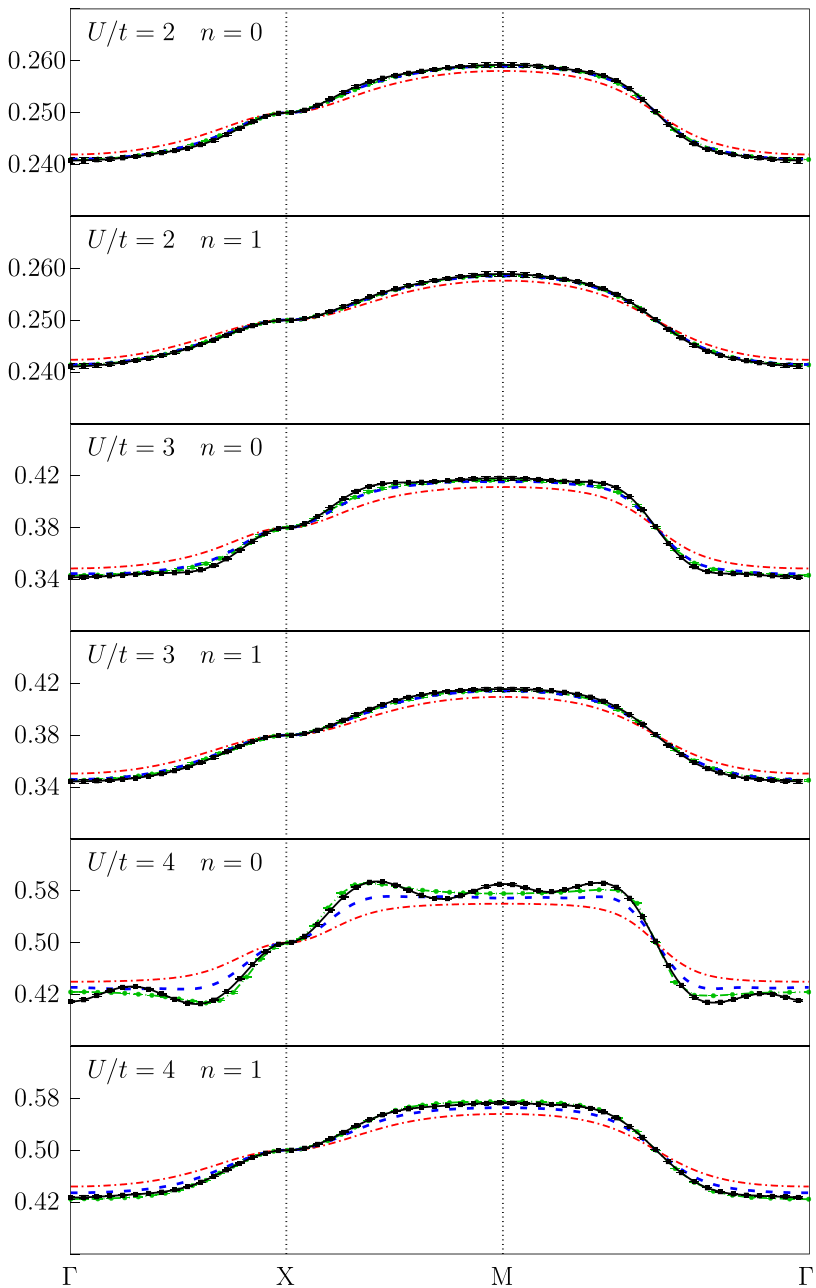

FIG. 6. Imaginary (left) and real (right) parts of the lattice self-energy $\Sigma(\mathbf{k})$ at the first and second Matsubara frequency $n=0,1$ along a path in the first Brillouin zone, for an inverse temperature $\beta t=5$ and different values of the interaction $U / t$. We plot the lattice self-energy obtained by conventional periodization of the $8 \times 8$ cluster (red), Fourier transformation of the real-space correlations relative to the central site for the $8 \times 8$ cluster (blue), Fourier transformation using the extrapolated real-space self-energy (black), and the numerically exact DiagMC data (green). 


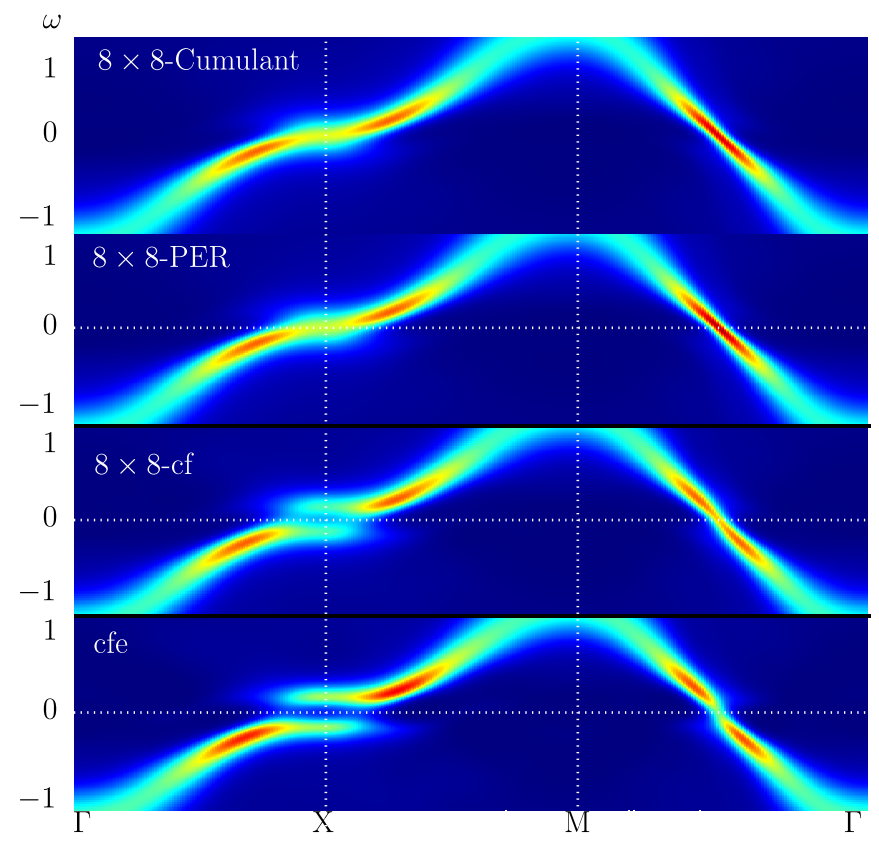

FIG. 7. Spectral function of the lattice Green's function, for $U / t=4.0$ and an inverse temperature $\beta t=5$, using the $8 \times 8 \mathrm{cu}-$ mulant periodization scheme, the conventional $8 \times 8$ periodization, the $8 \times 8$ center-focused reperiodization and the center-focused extrapolation (from top to bottom).

$U / t=4$, we observe a decay of the absolute value of the self-energy at the first Matsubara frequency to zero within less than 5 lattice constants.

In Fig. 6, we compare the Fourier transform

$$
\Sigma_{\text {latt }}\left(\mathbf{k}, i \omega_{n}\right)=\sum_{\mathbf{R}} \Sigma_{\mathbf{R}+\mathbf{R}_{\mathbf{c}}, \mathbf{R}_{\mathbf{c}}}^{N \rightarrow \infty}\left(i \omega_{n}\right) \mathrm{e}^{-\mathrm{ik} \cdot \mathbf{R}}
$$

of this estimator of the lattice self-energy (black) with the exact DiagMC computation (green) for the first two Matsubara frequencies $\left(\omega_{n=\{0,1\}}\right)$, at the temperature where it is available. We further compare these to the self-energy obtained by using the same procedure directly on the $N \times N=8 \times 8$ results, i.e., without the large $N$ extrapolation (blue dashed), and to the "standard" periodization of the $N \times N=8 \times 8$ self-energy (red) [20]. We find again an excellent agreement, within error bars, between the CDMFT+CFE and the exact DiagMC results. In particular, for the largest available $U$, we see a substantial improvement over the conventional self-energy periodization, and a sizable correction of the self-energy data obtained without extrapolation. We see in the second to lowest panel on the right side $(\beta t=5, U / t=4, n=0)$ that the CFE procedure captures even the nonmonotonous behavior of the self-energy along the $\Gamma-X-M-\Gamma$ path which is not present in the $N \times N=8 \times 8$ data. We remark that such fine structure in $\mathrm{k}$-space originates from cluster self-energy terms belonging to the largest distances $R$ whose calculation and error estimates are most challenging.

\section{Lattice spectral function of CDMFT+CFE}

The single particle spectra corresponding to the self-energy data of Fig. 6 are presented in Fig. 7. In addition, we present here also the spectrum for the cumulant periodization scheme applied to the $N \times N=8 \times 8$ cluster (see Appendix B).

The qualitative differences between the CFE results and the conventional schemes using data without extrapolation are sizable and most visible around the Fermi level. The gaps around $X=(0, \pi)$ and between $M$ and $\Gamma$ at $(\pi / 2, \pi / 2)$ are more pronounced due to the larger value of the CFE self-energies. This shows that the CFE has a qualitative impact on computed observables, even at high temperature.

\section{SPIN-SPIN CORRELATION FUNCTION}

Let us now consider the spin susceptibility of the cluster

$$
\begin{aligned}
\chi(\mathbf{R}, \tau) \equiv & \left\langle\left[\hat{n}_{\uparrow}\left(\mathbf{R}+\mathbf{R}_{\mathbf{c}}, \tau\right)-\hat{n}_{\downarrow}\left(\mathbf{R}+\mathbf{R}_{\mathbf{c}}, \tau\right)\right]\right. \\
& \left.\times\left[\hat{n}_{\uparrow}\left(\mathbf{R}_{\mathbf{c}}, 0\right)-\hat{n}_{\downarrow}\left(\mathbf{R}_{\mathbf{c}}, 0\right)\right]\right\rangle .
\end{aligned}
$$

The computation of the full lattice susceptibility, which uses the impurity two-particle vertex function as an input to the lattice Bethe-Salpeter equation [3], is currently computationally too demanding for large clusters. However, in the limit of infinite cluster size, we expect the two quantities to coincide. In Fig. 8 (upper panel), we show the imaginary time onsite and nearest-neighbor spin-spin correlation functions for $U / t=4$ and $\beta t=7.5$, for different cluster sizes. For these parameters, both components are (i) sizable, (ii) fully dynamic (i.e., beyond static mean-field approaches), and (iii) antiferromagnetic (as expected for the present model). We see a quick convergence of the onsite susceptibility with $N$. The nearest-neighbor component for $R=1$ has a stronger cluster dependence. In the central panel of Fig. 8, we show the spatially resolved dynamical susceptibility $\chi(\mathbf{R}, \tau)$ for the $8 \times 8$ cluster. Remarkably, the dynamic part of the susceptibility seems to decay much faster with distance than the static part [i.e., already $\chi(\mathbf{R}=(1,1), \tau)$ is barely dependent on $\tau$ ]. This could indicate the presence of two length scales, one for coherent (i.e., dynamic) and one for nonlocal correlations that can be captured by static mean fields. The real-space decay of the static (i.e., $\tau$-integrated) spin susceptibility $\chi(\mathbf{R}, \Omega=0)$ is presented on the bottom panel of Fig. 8 and compared to that of the corresponding self-energy at the smallest Matsubara frequency. The susceptibility data is well approximated by an Ornstein-Zernike

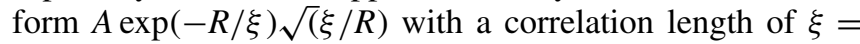
6.89. The fact that we manage to capture the exponential decay of spin-spin correlations reaching far beyond the scale of the clusters under consideration is indeed quite remarkable. A similar analysis for the self-energy proves to be less feasible, given the fact that it exhibits a much stronger radial dependence. We observe, however, that the self-energy decays overall on smaller length scales than the susceptibility.

In order to assess the validity of these susceptibility results, we compare it for different cluster sizes $N \times N=4 \times 4,6 \times$ $6,8 \times 8$ to the DiagMC benchmark in Fig. 9, where we plot $\chi\left(\mathbf{R}, i \Omega_{n}\right)$ as a function of Matsubara frequency for the two displacements $\mathbf{R}=(0,0)$ (left) and $\mathbf{R}=(1,0)$ (right). 

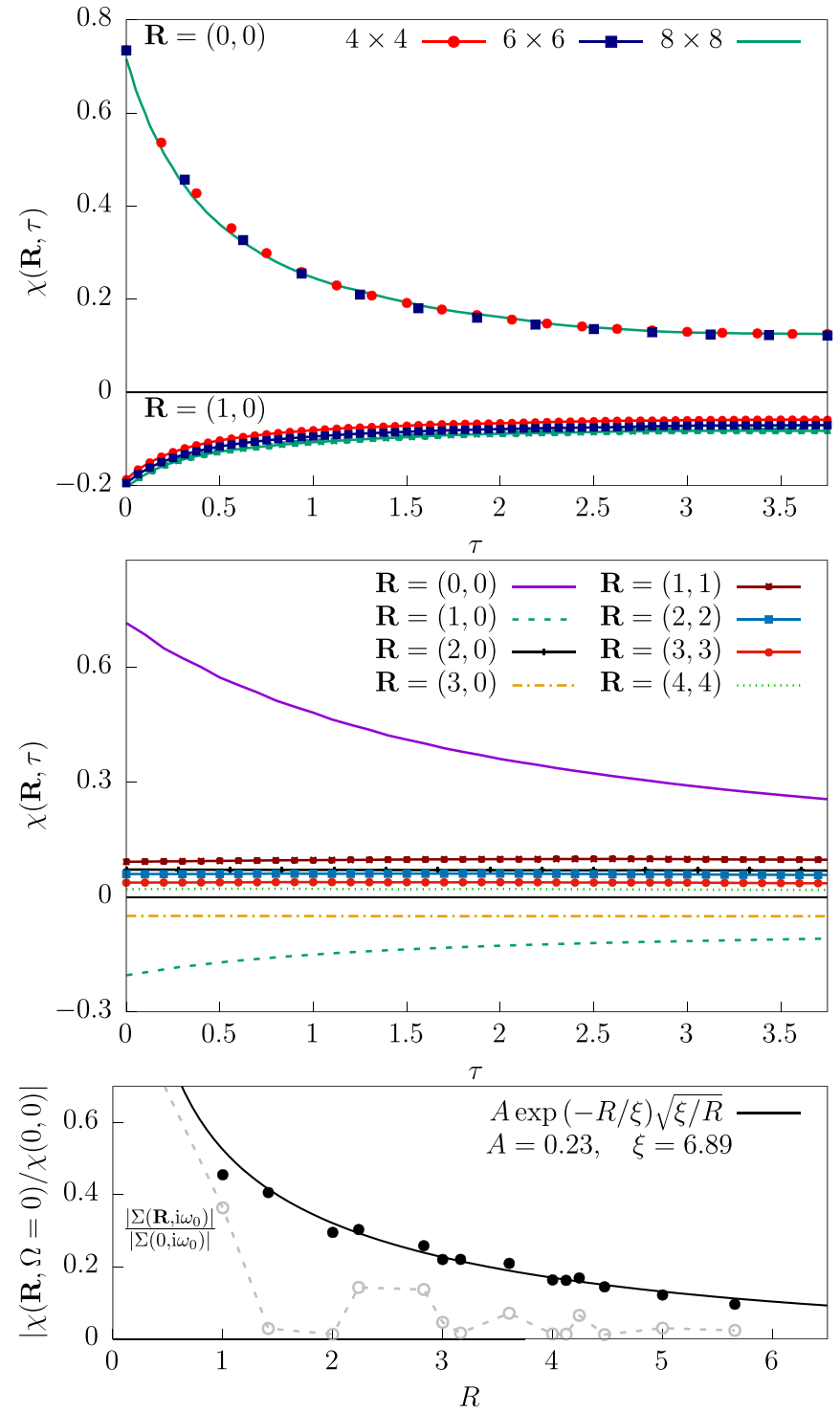

FIG. 8. (Top) Spin-spin correlation function $\chi(\mathbf{R}, \tau)$ as defined in Eq. 6 at distances $R=0$ and $R=1$, for $\beta t=7.5, U / t=4$, and three different cluster sizes. While the onsite component appears to be almost cluster-size independent, the nearest-neighbor one exhibits a dependence on $N$. (Middle) The same as the upper panel, but for fixed cluster size $N \times N=8 \times 8$ and various horizontal and diagonal displacement vectors. (Bottom) Normalized static spin susceptibility (colored) and self-energy (gray) as a function of the real-space distance $R$ for an $N \times N=8 \times 8$ cluster for the same displacement vectors as above. An exponential fit of the data is shown in black.

As for the self-energy, we perform an extrapolation to infinite cluster size (cf. insets of Fig. 9) and obtain excellent agreement, within error bars, with the DiagMC data. We observe a strong dependence on $N$ only for the displacement $\mathbf{R}=(1,0)$ at the first Matsubara frequency.

Our analysis of the spin-spin susceptibility suggests that real-space cluster approaches such as CDMFT are valid even at temperatures and/or interaction values for which the magnetic correlation length exceeds the cluster size, as long as the self-energy decay in real-space is sufficiently captured. However, we expect that the feedback of this long-range magnetic mode onto the electronic self-energy is not correctly described at this cluster size.

\section{CONCLUSION}

In this work, we have revisited the CDMFT calculations for the single band Hubbard model on the 2D square lattice at half filling. We performed a detailed momentum and real-space analysis of the spectral properties for different cluster sizes of up to $9 \times 9$ sites. Using a systematic benchmark with the exact DiagMC result at the lowest temperature for which it is obtainable, we have shown that an approximation scheme of the lattice self-energy based on the center of the cluster is superior to the conventional periodization approaches based on averages over the cluster. In fact, the broken translational symmetry of the cluster Green's function and self-energy is simply a manifestation of the bulk-like nature of the central cluster sites, making them the proper basis for the approximation of the respective lattice quantities. We have further shown that the exponential decay of spin-spin correlations is very well captured by CDMFT calculations, even when correlations extend far beyond the size of the cluster. Finally, CDMFT calculations and the CFE extrapolation can be carried out to temperatures currently inaccessible to exact diagrammatic Monte Carlo techniques, making the CDMFT+CFE procedure a powerful computational tool to access the physics of nonlocal correlations beyond dynamical mean-field theory. The presented algorithmic advancements may be applied to improve material-realistic studies of correlated electron systems such as transition metal oxides [75], molecular materials like the family of organic superconductors [76], and correlated ad-atoms on semiconductor surfaces [77-80]. Within practical restrictions, i.e., an upper limit for local (spin-orbital) plus cluster degrees of freedom, CDMFT+CFE can help to explore regimes in the phase diagram which are beyond the local limit such as second order phase transitions (e.g., magnetic ordering or superconducting pairing). Furthermore, for real material studies the computation of two-particle observables (as the dynamic magnetic susceptibility presented in this paper) allows for a connection with modern types of spectroscopies such as, e.g., x-ray absorption or (non)-resonant inelastic x-ray scattering which reveal phenomena of many-body correlations much less ambiguous than the single particle spectral function.

\section{ACKNOWLEDGMENTS}

The authors thank A. Georges, K. Held, J. LeBlanc, A. Toschi, G. Sangiovanni, and in particular, A.-M. Tremblay for very valuable discussions, and S. Heinzelmann and C. Hille for a critical reading of the manuscript. The Flatiron Institute is a division of the Simons Foundation. We acknowledge financial support from the Deutsche Forschungsgemeinschaft (DFG) through ZUK 63 and Project No. AN 815/6-1. The present work was also supported by the Austrian Science Fund (FWF) through the Erwin-Schrödinger Fellowship J 4266 "Superconductivity in the vicinity of Mott insulators" (SuMo, T.S.), as well as the European Research Council for the European Union Seventh Framework Program (FP7/2007-2013) 

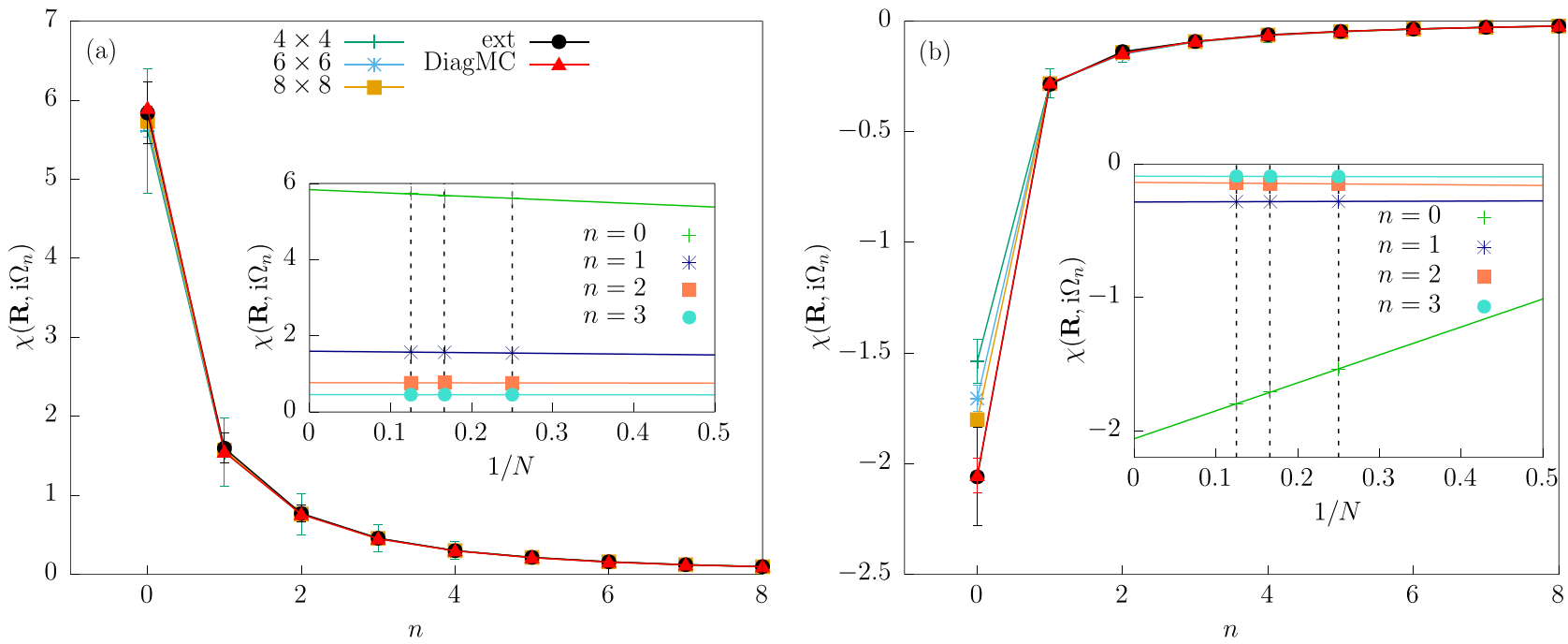

FIG. 9. Spin susceptibility for $\mathbf{R}=(0,0)$ (a) and $\mathbf{R}=(1,0)$ (b), as obtained from the various CDMFT clusters and DiagMC for $U / t=4$ and $\beta t=5$. The extrapolation is obtained by fitting the data with a $1 / N$ linear function, see insets for the fits from the first four Matsubara frequencies.

with ERC Grant No. 319286 (QMAC, T.S.) and the Simons Collaboration on the Many-Electron Problem. Calculations were partly performed on the cluster at the MPI for Solid State Research in Stuttgart. The authors acknowledge also the CPHT computer support team for their help.

\section{APPENDIX A: ANTIFERROMAGNETIC ORDER}

In this Appendix, we consider antiferromagnetic (AFM) ordering in our CDMFT computations.

In Fig. 10, we provide the phase boundary $T_{N}(U)$ as determined by the CDMFT with the criterion: AFM order for $m_{\mathrm{AFM}} \geqslant 0.1$ and $\mathrm{PM}$ one for $m_{\mathrm{AFM}} \leqslant 0.1$. We consider cluster sizes up to $8 \times 8$. Due to commensurability with the AFM checkerboard symmetry, we restrict our analysis to even $N$ (even though odd clusters could also be easily accessed in selfconsistent cycles analogous to AFM single site DMFT). We observe a monotonous reduction of the Néel temperature with increasing cluster size, with a pronounced $U$ dependence: the reduction of $T_{N}$ in the strong coupling regime is significantly stronger compared to the small $U$ region. This reduction is

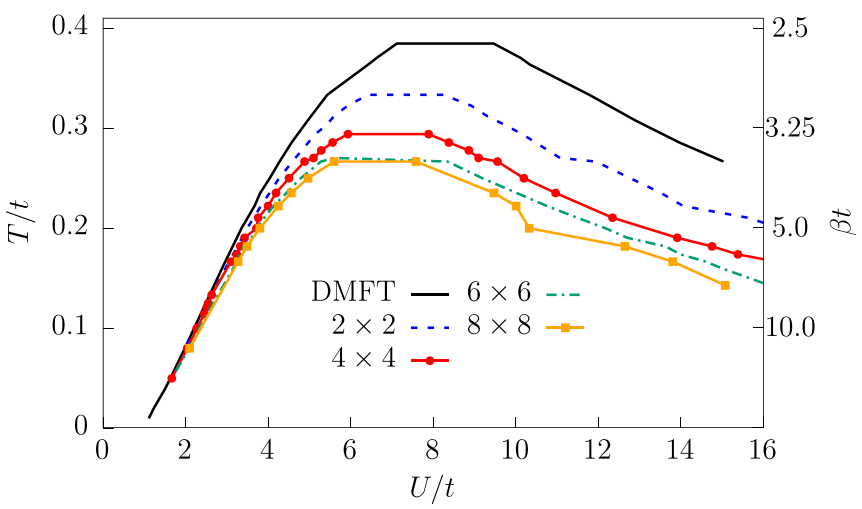

FIG. 10. Néel temperatures for a DMFT and various CDMFT calculations with even cluster sizes $N$. expected, and compatible with the exact Néel temperature of the $2 \mathrm{D}$ square lattice, which is known to be $T_{N}=0$ from the Mermin-Wagner theorem [81].

\section{APPENDIX B: REPERIODIZED SPECTRAL FUNCTIONS}

In this supplemental section, we present directly postprocessed CDMFT data without extrapolation for various cluster sizes. In order to compute momentum dependent spectral functions in the Brillouin zone of the 2D square lattice, we follow the cumulant periodization scheme of Ref. [26]. Instead of periodizing the self-energy directly, its cumulant $M\left(\mathrm{i} \omega_{n}\right)=\left(\mathrm{i} \omega_{n}+\mu-\Sigma\left(\mathrm{i} \omega_{n}\right)\right)^{-1}$ is periodized to obtain the lattice Green's function $G\left(\mathbf{k}, \mathrm{i} \omega_{n}\right)$ by

$$
M\left(\mathbf{k}, \mathrm{i} \omega_{n}\right)=\frac{1}{N} \sum_{i, j=1}^{N} M_{i, j}\left(\mathrm{i} \omega_{n}\right) \mathrm{e}^{\mathrm{i} \mathbf{k}\left(\mathbf{R}_{i}-\mathbf{R}_{j}\right)}
$$

with $\mathbf{R}_{i}, \mathbf{R}_{j}$ being the real-space positions of the sites $i$ and $j$ in the cluster, and the origin in $\mathbf{R}=(0,0)$ corresponding to the central site for $N$ odd and to one of the innermost sites for $N$ even. We note that also the application of this scheme turned out to be problematic in some situations-which actually motivates the present analysis to account for the real-space anisotropies of CDMFT_-but is used here as a reference since it was shown to perform better than reperiodizations of the self-energy or the Green's function [26].

In Fig. 11, we show results for the single-particle spectral function as obtained from CDMFT for $U / t=4$ and an inverse temperature $\beta t=12.5$ by employing (B1) and subsequent analytical continuation with the Maximum entropy method $[82,83]$. Each of the nine horizontal panels contains a momentum resolved intensity map for $A(\omega, \mathbf{k})$ (left) on the path $\Gamma-X-M-\Gamma$ in the cubic Brillouin zone and the $\mathbf{k}$-integrated local spectral function $A(\omega)$ (right). The evolution from top ("DMFT") to bottom ("9 $\times 9$ ") illustrates the cluster size dependence of the spectrum at fixed interaction and temperature. As function a of $N$, we observe an MIT transition which 


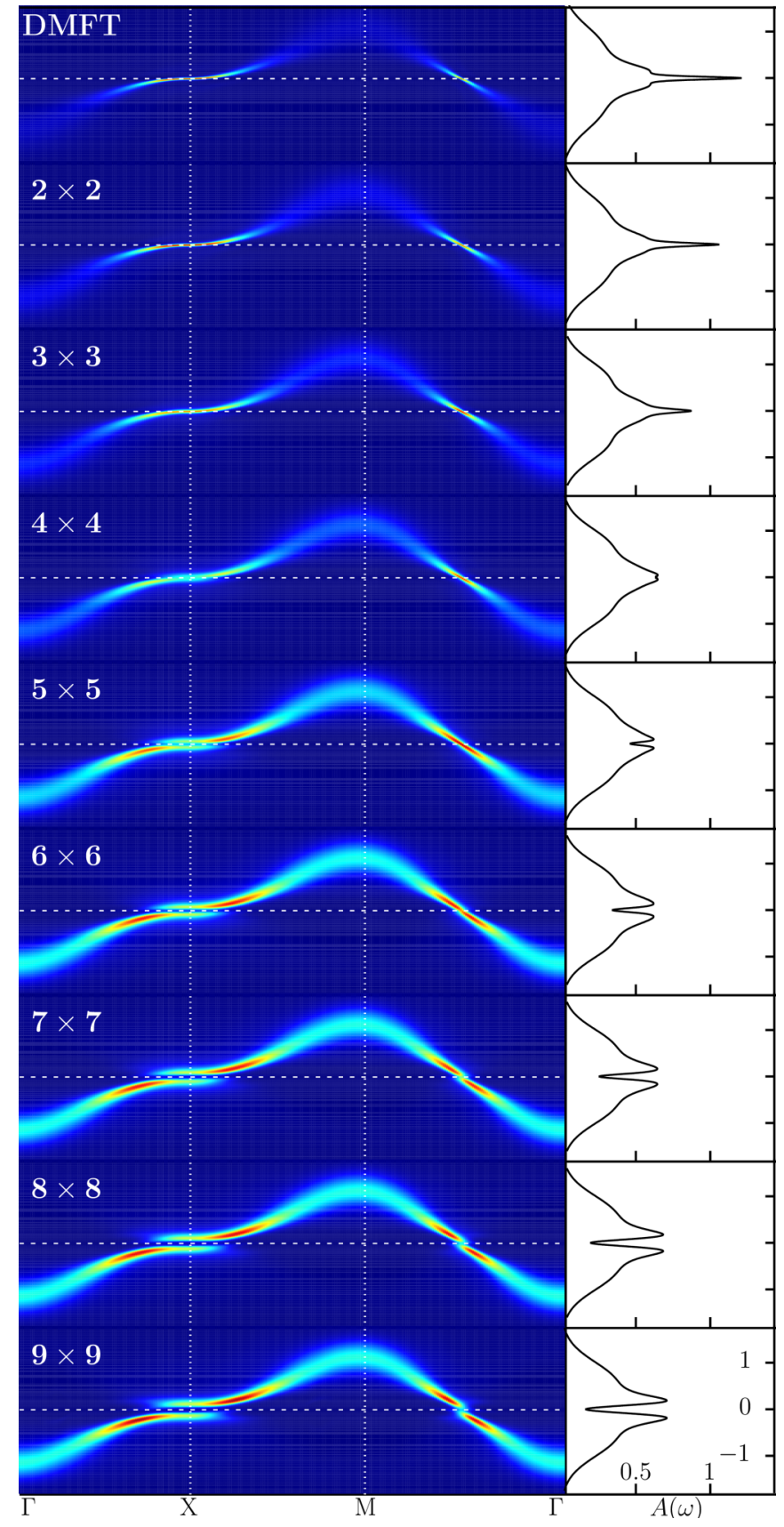

FIG. 11. Momentum resolved spectral functions along a closed path inside the first Brillouin zone (left) and full spectral function of the local Green's function (right), for $U / t=4$, inverse temperature $\beta t=12.5$, and different square clusters, as obtained by using the cumulant periodization. The dotted line corresponds to $\omega=0$. While the single-site DMFT $(N=1)$ is clearly in the metallic phase, the quasiparticle peak vanishes with increasing $N$, and $N \times N=$ $9 \times 9$ is already close to the insulating phase characterized by a gap in the local spectral function.

reflects in $A(\omega)$ as a spectral weight transfer from sharp quasiparticle excitations around the Fermi level $\varepsilon_{F}$ to incoherent Hubbard bands, in agreement with previous studies on smaller cluster sizes [24,27]. However, the opening of the gap in $A(\omega)$ is a gradual process crossing a pseudogap-like regime between $N \times N=4 \times 4$ and $N \times N=7 \times 7$. This effect originates

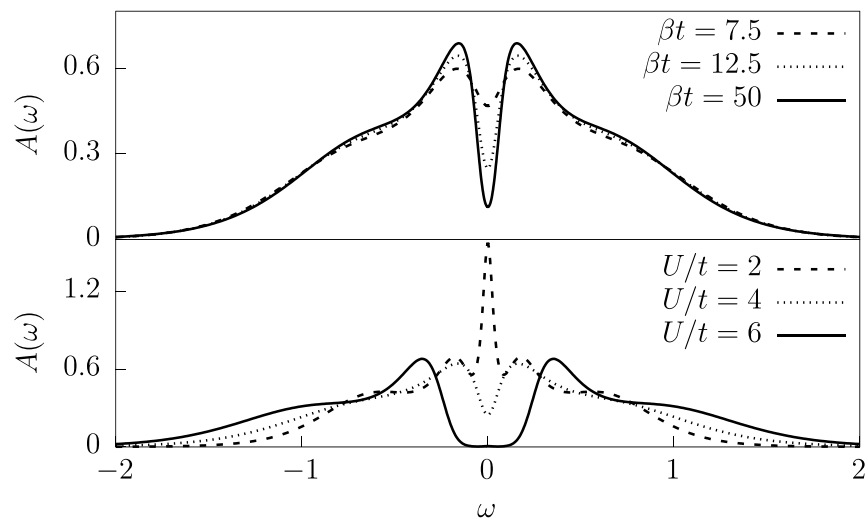

FIG. 12. Spectral function of the local lattice Green's function for $N \times N=7 \times 7$, for different inverse temperatures and fixed $U / t=4$ (top), and for different values of $U$ and a fixed inverse temperature $\beta t=12.5$ (bottom).

from a momentum selective opening of the gap (see also Ref. [84]). Closer inspection of $A(\omega, \mathbf{k})$ for the intermediate regime of $N \times N=5 \times 5$ and $6 \times 6$ clearly reveals the absence of a Fermi surface at the antinodal point $X=(\pi, 0)$, while the quasiparticle band crossing $\varepsilon_{F}$ in the nodal direction around $(\pi / 2, \pi / 2)$ remains sharp.

To complement our analysis of the MIT we also study the $U$ and $\beta$ dependencies of $A(\omega)$. Figure 12 shows $A(\omega)$ for $N \times N=7 \times 7$ and $U / t=4$. The temperature dependence, especially of the spectral weight at $\varepsilon_{F}$, indicates the presence of a fully developed gap (in agreement with the corresponding $N \times N=7 \times 7$ momentum resolved spectrum) which is barely closed by incoherent thermal smearing. Data without analytical continuation are reported in the lower panel of Fig. 13, with $G(\tau=\beta / 2)=A(\omega=0)$.

Here our results for the MIT as a function of $N, U$, and temperature are summarized. Remarkably, the slope of the $T_{c}$

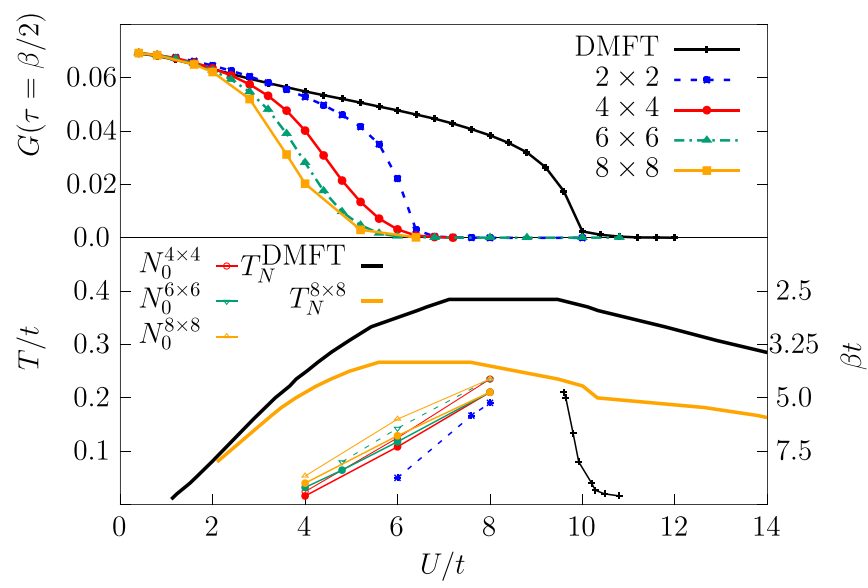

FIG. 13. Weight of the imaginary time local lattice Green's function evaluated at $\tau=\beta / 2$, for a fixed inverse temperature $\beta t=12.5$ and different cluster sizes (top) and phase diagram with the Mott transition line for different cluster sizes (bottom). For comparison, we show also the transition lines as obtained from the local lattice Green's function (dashed lines). The Néel temperatures for the smallest and largest cluster size is indicated by dotted lines. 
versus $U_{c}$ transition line is inverted with respect to the singlesite DMFT already for the smallest $N \times N=2 \times 2$ cluster, in agreement with previous works [24]. Increasing $N$ leads to a simultaneous increase of $T_{c}$ at fixed $U$ and decrease of
$U_{c}$ at fixed $T$ which is consistent with previous calculations directly in the thermodynamic limit, e.g., DГA $[85,86]$ and DiagMC [69,87], where the critical value shows the behavior $U_{c} \rightarrow 0$.
[1] W. Metzner and D. Vollhardt, Phys. Rev. Lett. 62, 324 (1989).

[2] A. Georges and G. Kotliar, Phys. Rev. B 45, 6479 (1992).

[3] A. Georges, G. Kotliar, W. Krauth, and M. J. Rozenberg, Rev. Mod. Phys. 68, 13 (1996).

[4] G. Kotliar, S. Y. Savrasov, K. Haule, V. S. Oudovenko, O. Parcollet, and C. A. Marianetti, Rev. Mod. Phys. 78, 865 (2006).

[5] P. Hansmann, A. Toschi, G. Sangiovanni, T. Saha-Dasgupta, S. Lupi, M. Marsi, and K. Held, Phys. Status Solidi (b) 250, 1251 (2013).

[6] J. Bednorz and K. Müller, Z. Phys. B: Condens. Matter 64, 189 (1986).

[7] E. Dagotto, Rev. Mod. Phys. 66, 763 (1994).

[8] T. Timusk and B. Statt, Rep. Prog. Phys. 62, 61 (1999).

[9] P. A. Lee, N. Nagaosa, and X.-G. Wen, Rev. Mod. Phys. 78, 17 (2006).

[10] A. Tamai, M. Zingl, E. Rozbicki, E. Cappelli, S. Riccò, A. de la Torre, S. McKeown Walker, F. Y. Bruno, P. D. C. King, W. Meevasana, M. Shi, M. Radović, N. C. Plumb, A. S. Gibbs, A. P. Mackenzie, C. Berthod, H. U. R. Strand, M. Kim, A. Georges, and F. Baumberger, Phys. Rev. X 9, 021048 (2019).

[11] H. v. Löhneysen, A. Rosch, M. Vojta, and P. Wölfle, Rev. Mod. Phys. 79, 1015 (2007).

[12] N. Büttgen, H. Krug von Nidda, W. Kraetschmer, A. Günther, S. Widmann, S. Riegg, A. Krimmel, and A. Loidl, J. Low Temp. Phys. 161, 148 (2010).

[13] P. Gegenwart, Q. Si, and F. Steglich, Nat. Phys. 4, 186 (2008).

[14] T. Schäfer, N. Wentzell, F. Šimkovic IV, Y.-Y. He, C. Hille, M. Klett, C. J. Eckhardt, B. Arzhang, V. Harkov, F.-M. L. Régent, A. Kirsch, Y. Wang, A. J. Kim, E. Kozik, E. A. Stepanov, A. Kauch, S. Andergassen, P. Hansmann, D. Rohe, Y. M. Vilk, J. P. F. LeBlanc, S. Zhang, A. M. S. Tremblay, M. Ferrero, O. Parcollet, and A. Georges, arXiv:2006.10769.

[15] M. H. Hettler, A. N. Tahvildar-Zadeh, M. Jarrell, T. Pruschke, and H. R. Krishnamurthy, Phys. Rev. B 58, R7475 (1998).

[16] M. H. Hettler, M. Mukherjee, M. Jarrell, and H. R. Krishnamurthy, Phys. Rev. B 61, 12739 (2000).

[17] K. Aryanpour, M. H. Hettler, and M. Jarrell, Phys. Rev. B 65, 153102 (2002).

[18] T. A. Maier, M. Jarrell, T. Prushke, and M. Hettler, Rev. Mod. Phys. 77, 1027 (2005).

[19] A. I. Lichtenstein and M. I. Katsnelson, Phys. Rev. B 62, R9283 (2000).

[20] G. Kotliar, S. Y. Savrasov, G. Pálsson, and G. Biroli, Phys. Rev. Lett. 87, 186401 (2001).

[21] A. Liebsch, H. Ishida, and J. Merino, Phys. Rev. B 78, 165123 (2008).

[22] G. Biroli, O. Parcollet, and G. Kotliar, Phys. Rev. B 69, 205108 (2004).

[23] J. Vučičević, N. Wentzell, M. Ferrero, and O. Parcollet, Phys. Rev. B 97, 125141 (2018).

[24] O. Parcollet, G. Biroli, and G. Kotliar, Phys. Rev. Lett. 92, 226402 (2004).
[25] T. D. Stanescu, M. Civelli, K. Haule, and G. Kotliar, Ann. Phys. 321, 1682 (2006).

[26] S. Sakai, G. Sangiovanni, M. Civelli, Y. Motome, K. Held, and M. Imada, Phys. Rev. B 85, 035102 (2012).

[27] H. Park, K. Haule, and G. Kotliar, Phys. Rev. Lett. 101, 186403 (2008).

[28] L. Fratino, P. Sémon, M. Charlebois, G. Sordi, and A. M. S. Tremblay, Phys. Rev. B 95, 235109 (2017).

[29] C. Walsh, P. Sémon, D. Poulin, G. Sordi, and A. M. S. Tremblay, Phys. Rev. Lett. 122, 067203 (2019).

[30] C. Walsh, P. Sémon, D. Poulin, G. Sordi, and A. M. S Tremblay, Phys. Rev. B 99, 075122 (2019).

[31] A. Foley, S. Verret, A. M. S. Tremblay, and D. Sénéchal, Phys. Rev. B 99, 184510 (2019).

[32] J. Vučičević, J. Kokalj, R. Žitko, N. Wentzell, D. Tanasković, and J. Mravlje, Phys. Rev. Lett. 123, 036601 (2019).

[33] M. Civelli, M. Capone, A. Georges, K. Haule, O. Parcollet, T. D. Stanescu, and G. Kotliar, Phys. Rev. Lett. 100, 046402 (2008).

[34] M. Ferrero, O. Parcollet, A. Georges, G. Kotliar, and D. N. Basov, Phys. Rev. B 82, 054502 (2010).

[35] E. Gull, O. Parcollet, and A. J. Millis, Phys. Rev. Lett. 110, 216405 (2013).

[36] T. A. Maier, M. Jarrell, A. Macridin, and C. Slezak, Phys. Rev. Lett. 92, 027005 (2004)

[37] T. A. Maier, M. Jarrell, T. C. Schulthess, P. R. C. Kent, and J. B. White, Phys. Rev. Lett. 95, 237001 (2005).

[38] E. Gull, M. Ferrero, O. Parcollet, A. Georges, and A. J. Millis, Phys. Rev. B 82, 155101 (2010).

[39] S.-X. Yang, H. Fotso, S.-Q. Su, D. Galanakis, E. Khatami, J.-H. She, J. Moreno, J. Zaanen, and M. Jarrell, Phys. Rev. Lett. 106, 047004 (2011).

[40] S. Okamoto, D. Sénéchal, M. Civelli, and A. M. S. Tremblay, Phys. Rev. B 82, 180511(R) (2010).

[41] M. Ferrero, P. S. Cornaglia, L. D. Leo, O. Parcollet, G. Kotliar, and A. Georges, Europhys. Lett. 85, 57009 (2009).

[42] A. Toschi, A. A. Katanin, and K. Held, Phys. Rev. B 75, 045118 (2007).

[43] A. A. Katanin, A. Toschi, and K. Held, Phys. Rev. B 80, 075104 (2009).

[44] A. Valli, G. Sangiovanni, O. Gunnarsson, A. Toschi, and K. Held, Phys. Rev. Lett. 104, 246402 (2010).

[45] G. Rohringer, A. Toschi, A. Katanin, and K. Held, Phys. Rev. Lett. 107, 256402 (2011).

[46] A. N. Rubtsov, M. I. Katsnelson, and A. I. Lichtenstein, Phys. Rev. B 77, 033101 (2008).

[47] H. Hafermann, G. Li, A. N. Rubtsov, M. I. Katsnelson, A. I. Lichtenstein, and H. Monien, Phys. Rev. Lett. 102, 206401 (2009).

[48] T. Ayral and O. Parcollet, Phys. Rev. B 92, 115109 (2015).

[49] T. Ayral and O. Parcollet, Phys. Rev. B 93, 235124 (2016).

[50] T. Ayral and O. Parcollet, Phys. Rev. B 94, 075159 (2016). 
[51] J. Vučičević, T. Ayral, and O. Parcollet, Phys. Rev. B 96, 104504 (2017).

[52] T. Ayral, J. Vučičević, and O. Parcollet, Phys. Rev. Lett. 119, 166401 (2017).

[53] G. Rohringer, A. Toschi, H. Hafermann, K. Held, V. I. Anisimov, and A. A. Katanin, Phys. Rev. B 88, 115112 (2013).

[54] J. P. Hague, M. Jarrell, and T. C. Schulthess, Phys. Rev. B 69, 165113 (2004).

[55] C. Slezak, M. Jarrell, T. Maier, and J. Deisz, J. Phys.: Condens. Matter 21, 435604 (2009).

[56] S.-X. Yang, H. Fotso, H. Hafermann, K.-M. Tam, J. Moreno, T. Pruschke, and M. Jarrell, Phys. Rev. B 84, 155106 (2011).

[57] C. Taranto, S. Andergassen, J. Bauer, K. Held, A. Katanin, W. Metzner, G. Rohringer, and A. Toschi, Phys. Rev. Lett. 112, 196402 (2014).

[58] G. Rohringer, H. Hafermann, A. Toschi, A. A. Katanin, A. E. Antipov, M. I. Katsnelson, A. I. Lichtenstein, A. N. Rubtsov, and K. Held, Rev. Mod. Phys. 90, 025003 (2018).

[59] W. Wu, M. Ferrero, A. Georges, and E. Kozik, Phys. Rev. B 96, 041105(R) (2017).

[60] J. P. F. LeBlanc, A. E. Antipov, F. Becca, I. W. Bulik, G. K.-L. Chan, C.-M. Chung, Y. Deng, M. Ferrero, T. M. Henderson, C. A. Jiménez-Hoyos, E. Kozik, X.-W. Liu, A. J. Millis, N. V. Prokof'ev, M. Qin, G. E. Scuseria, H. Shi, B. V. Svistunov, L. F. Tocchio, I. S. Tupitsyn, S. R. White, S. Zhang, B.-X. Zheng, Z. Zhu, and E. Gull (Simons Collaboration on the Many-Electron Problem), Phys. Rev. X 5, 041041 (2015).

[61] T. D. Stanescu and G. Kotliar, Phys. Rev. B 74, 125110 (2006).

[62] D. Sénéchal, D. Perez, and M. Pioro-Ladrière, Phys. Rev. Lett. 84, 522 (2000).

[63] N. V. Prokof'ev and B. V. Svistunov, Phys. Rev. Lett. 81, 2514 (1998).

[64] K. Van Houcke, E. Kozik, N. Prokof'ev, and B. Svistunov, Phys. Procedia 6, 95 (2010).

[65] E. Kozik, K. Van Houcke, E. Gull, L. Pollet, N. Prokof'ev, B. Svistunov, and M. Troyer, Europhys. Lett. 90, 10004 (2010).

[66] R. Rossi, Phys. Rev. Lett. 119, 045701 (2017).

[67] A. Moutenet, W. Wu, and M. Ferrero, Phys. Rev. B 97, 085117 (2018).

[68] F. Šimkovic and E. Kozik, Phys. Rev. B 100, 121102(R) (2019).
[69] F. Šimkovic, J. P. F. LeBlanc, A. J. Kim, Y. Deng, N. V. Prokof'ev, B. V. Svistunov, and E. Kozik, Phys. Rev. Lett. 124, 017003 (2020).

[70] A. N. Rubtsov and A. I. Lichtenstein, J. Exp. Theor. Phys. Lett. 80, 61 (2004).

[71] O. Parcollet, M. Ferrero, T. Ayral, H. Hafermann, I. Krivenko, L. Messio, and P. Seth, Comput. Phys. Commun. 196, 398 (2015).

[72] S. Sakai, Y. Motome, and M. Imada, Phys. Rev. Lett. 102, 056404 (2009).

[73] P. Staar, T. Maier, and T. C. Schulthess, Phys. Rev. B 88, 115101 (2013).

[74] S. Sakai, M. Civelli, and M. Imada, Phys. Rev. Lett. 116, 057003 (2016).

[75] M. Imada, A. Fujimori, and Y. Tokura, Rev. Mod. Phys. 70, 1039 (1998).

[76] M. Dressel and N. Drichko, Chem. Rev. 104, 5689 (2004).

[77] E. Tosatti and P. W. Anderson, Jpn. J. Appl. Phys. 13, 381 (1974).

[78] G. Profeta and E. Tosatti, Phys. Rev. Lett. 98, 086401 (2007).

[79] S. Schuwalow, D. Grieger, and F. Lechermann, Phys. Rev. B 82, 035116 (2010).

[80] X. Cao, T. Ayral, Z. Zhong, O. Parcollet, D. Manske, and P. Hansmann, Phys. Rev. B 97, 155145 (2018).

[81] N. D. Mermin and H. Wagner, Phys. Rev. Lett. 17, 1133 (1966).

[82] R. K. Skilling, and J. Bryan, Mon. Not. R. Astron. Soc. 211, 111 (1984).

[83] G. J. Kraberger, R. Triebl, M. Zingl, and M. Aichhorn, Phys. Rev. B 96, 155128 (2017).

[84] D. Rost, E. V. Gorelik, F. Assaad, and N. Blümer, Phys. Rev. B 86, 155109 (2012).

[85] T. Schäfer, F. Geles, D. Rost, G. Rohringer, E. Arrigoni, K. Held, N. Blümer, M. Aichhorn, and A. Toschi, Phys. Rev. B 91, 125109 (2015).

[86] T. Schäfer, A. Toschi, and K. Held, J. Magn. Magn. Mater. 400, 107 (2016).

[87] A. J. Kim, F. Simkovic, and E. Kozik, Phys. Rev. Lett. 124, 117602 (2020).

[88] S. R. White, D. J. Scalapino, R. L. Sugar, E. Y. Loh, J. E. Gubernatis, and R. T. Scalettar, Phys. Rev. B 40, 506 (1989). 